\title{
Osteoanabolic effect of alendronate and zoledronate on bone marrow stromal cells (BMSCs) isolated from aged female osteoporotic patients and its implications for their mode of action in the treatment of age-related bone loss
}

\author{
Lindtner, R A ; Tiaden, André N ; Genelin, K ; Ebner, H L ; Manzl, C ; Klawitter, Marina ; Sitte, I ; \\ von Rechenberg, Brigitte ; Blauth, M ; Richards, Peter
}

\begin{abstract}
In the present study, we evaluated the potential for aminobisphosphonates to enhance the development of bone-forming osteoblasts from progenitor cells isolated from aged female osteoporotic patients. The aminobisphosphonates tested significantly enhanced osteoblast formation and thus lend further insights into their possible mode of action in the treatment of osteoporosis. INTRODUCTION: The primary aim of this study was to evaluate the influence of aminobisphosphonates on the osteogenesis of human bone marrow stromal cells (hBMSCs) and mineralization of differentiating bone-forming cells isolated from osteoporotic patients. METHODS: The influence of aminobisphosphonate treatment on hBMSC osteogenesis was assessed by the quantitative measurement of alkaline phosphatase (ALP) activity, in addition to quantitative reverse transcription polymerase chain reaction and Western blot analysis of known osteogenic markers. Mineralized matrix formation by hBMSC-derived osteoblasts was visualized and quantified using Alizarin red staining. RESULTS: hBMSC cultures treated with osteogenic medium supplemented with zoledronate demonstrated a significant increase in Alizarin red staining after 3 weeks as compared to cells cultured in osteogenic medium alone. Similarly, cultures of differentiating hBMSCs isolated from patients receiving alendronate treatment also demonstrated an increased propensity for mineralization, even in the absence of further in vitro stimulation by zoledronate. The stimulatory effects of aminobisphosphonate treatment on hBMSC-derived osteoblast-mediated mineralization were independent of any alterations in ALP activity, although significant decreases in the expression levels of osteopontin (SPP1) were evident in hBMSCs following exposure to aminobisphosphonates. Further analysis including Western blotting and loss-of-function studies revealed osteopontin as having a negative influence on the mineralization of differentiating osteoporotic bone-forming cells. CONCLUSIONS: The results presented here demonstrate for the first time that aminobisphosphonate treatment of osteoporotic hBMSCs enhances their capacity for osteoblast formation and subsequent mineral deposition, thus supporting the concept of aminobisphosphonates as having an osteoanabolic effect in osteoporosis.
\end{abstract}

DOI: https://doi.org/10.1007/s00198-013-2494-3

Posted at the Zurich Open Repository and Archive, University of Zurich

ZORA URL: https://doi.org/10.5167/uzh-89518

Journal Article

Accepted Version

Originally published at: 
Lindtner, R A; Tiaden, André N; Genelin, K; Ebner, H L; Manzl, C; Klawitter, Marina; Sitte, I; von Rechenberg, Brigitte; Blauth, M; Richards, Peter (2014). Osteoanabolic effect of alendronate and zoledronate on bone marrow stromal cells (BMSCs) isolated from aged female osteoporotic patients and its implications for their mode of action in the treatment of age-related bone loss. Osteoporosis International, 25(3):1151-1161.

DOI: https://doi.org/10.1007/s00198-013-2494-3 
Draft Manuscript for Review

\section{Osteoanabolic effect of alendronate and zoledronate on bone marrow stromal cells (BMSCs) isolated from aged female osteoporotic patients and its implications for their mode of action in the treatment of age-related bone loss.}

\begin{tabular}{|c|c|}
\hline Journal: & Osteoporosis International \\
\hline Manuscript ID: & OI-2013-02-0146.R1 \\
\hline Manuscript Type: & Original Article \\
\hline Date Submitted by the Author: & $\mathrm{n} / \mathrm{a}$ \\
\hline Complete List of Authors: & $\begin{array}{l}\text { Lindtner, Richard; Innsbruck Medical University, Department of Trauma } \\
\text { Surgery and Sports Medicine } \\
\text { Tiaden, André; Zurich University, Bone and Stem Cell Research Group, } \\
\text { CABMM } \\
\text { Genelin, Konstantin; Innsbruck Medical University, Department of Trauma } \\
\text { Surgery and Sports Medicine } \\
\text { Ebner, Hannes; Innsbruck Medical University, Department of Trauma } \\
\text { Surgery and Sports Medicine } \\
\text { Manzl, Claudia; Innsbruck Medical University, Division of Developmental } \\
\text { Immunology } \\
\text { Klawitter, Marina; Zurich University, Bone and Stem Cell Research Group, } \\
\text { CABMM } \\
\text { Sitte, Ingrid; Innsbruck Medical University, Department of Trauma Surgery } \\
\text { and Sports Medicine } \\
\text { von Rechenberg, Brigitte; Zurich University, Musculoskeletal Research Unit, } \\
\text { CABMM } \\
\text { Blauth, Michael; Innsbruck Medical University, Department of Trauma } \\
\text { Surgery and Sports Medicine } \\
\text { Richards, Peter; Zurich University, Bone and Stem Cell Research Group, } \\
\text { CABMM }\end{array}$ \\
\hline Keyword: & $\begin{array}{l}\text { Osteoporosis, Aminobisphosphonate, Bone marrow stromal cell, Osteogenic } \\
\text { differentiation, Mineralization, Osteopontin }\end{array}$ \\
\hline
\end{tabular}

\section{SCHOLARONE \\ Manuscripts}


Osteoanabolic effect of alendronate and zoledronate on bone marrow stromal cells (BMSCs) isolated from aged female osteoporotic patients and its implications for their mode of action in the treatment of age-related bone loss.

Richard A. Lindtner ${ }^{\mathrm{a}^{*}}$, André N. Tiaden ${ }^{\mathrm{b}}$, Konstantin Genelin ${ }^{\mathrm{a}}$, Hannes L. Ebner ${ }^{\mathrm{a}}$, Claudia Manzl $^{\mathrm{c}}$, Marina Klawitter ${ }^{\mathrm{b}}$, Ingrid Sitte ${ }^{\mathrm{a}}$, Brigitte von Rechenberg ${ }^{\mathrm{d}}$, Michael Blauth ${ }^{\mathrm{a}}$, Peter J. Richards ${ }^{\mathrm{b}, \mathrm{e}}$

\begin{abstract}
${ }^{a}$ Department of Trauma Surgery and Sports Medicine, Innsbruck Medical University, Innsbruck, Austria. ${ }^{\mathrm{b}}$ Bone and Stem Cell Research Group, CABMM, University of Zurich, Switzerland. ${ }^{\mathrm{c}}$ Division of Developmental Immunology, Biocenter, Innsbruck Medical University, Innsbruck, Austria. ${ }^{\mathrm{d}}$ Musculoskeletal Research Unit, CABMM, University of Zurich, Switzerland. ${ }^{\mathrm{e}}$ Zurich Center for Integrative Human Physiology (ZIHP), University of Zurich, Switzerland. *both authors contributed equally to this study.
\end{abstract}

Corresponding Author: Dr. Peter J. Richards, CABMM, University of Zurich, Winterthurerstrasse 190, CH-8057 Zürich, Switzerland, Tel: +41-44-635-3801; $\quad$ Fax: +41-44-635-6840; peter.richards@cabmm.uzh.ch 


\begin{abstract}
Purpose The primary aim of this study was to evaluate the influence of aminobisphosphonates on the osteogenesis of hBMSCs and mineralization of differentiating bone-forming cells isolated from osteoporotic patients.

Methods The influence of aminobisphosphonate treatment on hBMSC osteogenesis was assessed by the quantitative measurement of alkaline phosphatase (ALP) activity, in addition to qRT-PCR and Western blot analysis of known osteogenic markers. Mineralized matrix formation by hBMSC-derived osteoblasts was visualized and quantified using Alizarin red staining.

Results hBMSC cultures treated with osteogenic medium supplemented with zoledronate demonstrated a significant increase in Alizarin red staining after 3 weeks as compared to cells cultured in osteogenic medium alone. Similarly, cultures of differentiating hBMSCs isolated from patients receiving alendronate treatment also demonstrated an increased propensity for mineralization, even in the absence of further in vitro stimulation by zoledronate. The stimulatory effects of aminobisphosphonate treatment on hBMSCderived osteoblast formation were independent of any alterations in ALP activity, although significant changes in the expression levels of osteopontin (SPPl) were evident in hBMSCs following exposure to aminobisphosphonates. Further analysis revealed osteopontin as having a negative influence on the mineralization of differentiating osteoporotic bone-forming cells.
\end{abstract}

Conclusions The results presented here demonstrate for the first time that aminobisphosphonate treatment of osteoporotic hBMSCs enhances their capacity for osteoblast formation and subsequent mineral deposition, thus supporting the concept of aminobisphosphonates as having an osteoanabolic effect in osteoporosis.

Keywords: Osteoporosis; Aminobisphosphonate; Bone marrow stromal cell; Osteogenic differentiation; mineralization. 


\begin{abstract}
Mini Abstract
In the present study, we evaluated the potential for aminobisphosphonates to enhance the development of bone-forming osteoblasts from progenitor cells isolated from aged female osteoporotic patients. The aminobisphosphonates tested significantly enhanced osteoblast formation and thus lend further insights into their possible mode of action in the treatment of osteoporosis.
\end{abstract}




\section{Introduction}

Age-related bone loss results from an imbalance between bone resorption and bone formation due to a spatially or temporally uncoupled activity of bone resorbing osteoclasts and bone forming osteoblasts [1]. The latter are derived from progenitor cells within the bone marrow termed bone marrow stromal cells (BMSCs), through a process of osteogenic differentiation. The ability to produce a sufficient number of functionally active osteoblasts is pivotal to preserving bone mass and represents an essential step in the continuous, complex and well orchestrated process of bone remodeling [2]. There is now a growing body of evidence to suggest that BMSCs are critically involved in the pathogenesis of osteoporosis [3-9], thus implying that the structural abnormalities associated with osteoporotic bone maybe as a consequence of inadequacies in bone cell differentiation. This concept is further supported by findings from studies investigating the role of BMSCs in experimental models of osteoporosis and ageing. Results from our own studies, as well as research performed by others, have shown that the osteoporotic-like phenotype observed in senescence-accelerated mouse prone 6 strain (SAMP6) mice is associated with deficits in the osteogenic differentiation potential of resident BMSCs [10-11]. Similarly, impaired BMSC osteogenesis has been observed in several transgenic mouse models in which many of the characteristic features of senile osteoporosis are evident [12-15]. Clearly therefore, stimulation of resident BMSC populations to undergo osteogenic differentiation through therapeutic intervention may be key to the success of treating osteoporosis in aged individuals.

The majority of current treatments available for osteoporosis are directed towards preventing bone resorption, with bisphosphonate therapy being the most widely used anti-resorptive approach to enhance bone strength in osteoporotic patients [16]. Bisphosphonates are a well-characterized class of synthetic compounds structurally related to pyrophosphate, and are thought to mediate their antiresorptive actions primarily through inhibition of osteoclast activity [17]. However, there are now an increasing number of reports alluding to the idea that the preventative effects of bisphosphonates on bone loss, maybe additionally mediated through their anabolic effects on cells of the osteoblastic lineage. In the majority of 
cases, both amino- and non-aminobisphosphonates have been found to enhance osteogenic differentiation of human BMSCs (hBMSCs) [18-21]. These observations have also been extended to human adiposederived stromal cells (hASCs), where alendronate treatment not only stimulated osteogenesis in vitro, but also enhanced bone repair in a rat critical-sized calvarial defect model when delivered locally on a hASC/PLGA scaffold construct [22]. However, despite these observations, no study has yet examined the effects of bisphosphonates on hBMSCs isolated from osteoporotic patients, even though they are the primary target group for bisphosphonate treatment. The need for such investigations is further highlighted by the fact that hBMSCs from osteoporotic patients are considered to be molecularly distinct from their non-osteoporotic counterparts and may therefore not respond in the same way to bisphosphonate treatment [23].

In the present study, we aim to address this issue by evaluating the potential of aminobisphosphonates to enhance osteogenic differentiation of hBMSCs isolated from aged female osteoporotic patients. In vitro cultures of hBMSCs were incubated with zoledronate and their ability to differentiate towards osteoblasts assessed through the use of molecular, biochemical and histological techniques. Furthermore, the osteogenic differentiation potential of hBMSCs isolated from osteoporotic patients undergoing alendronate therapy was also evaluated. We hypothesized that exposure of osteoporotic hBMSCs to aminobisphosphonate would significantly increase their capacity for osteoblastogenesis and subsequent mineralization.

\section{Materials and methods}

\section{Patients}

BMSCs were isolated from a total of 10 aged female osteoporotic donors undergoing routine surgical procedures for proximal femur fracture at the Department of Trauma Surgery, University Hospital Innsbruck, Austria (Table 1). All procedures were carried out in accordance with the ethics commission guidelines for Innsbruck Medical University. Inclusion criteria consisted of patients being $>65$ years of 
age and having sustained a proximal femoral fracture after a low-energy trauma. Osteoporosis was confirmed in these patients using dual energy X-ray absorptiometry (DXA; Hologic QDR 4500) where those patients showing a bone mineral density T-score of -2.5 standard deviations (SD) or less at the contralateral hip and/or lumbar spine were considered osteoporotic. In three of the cases, patients had been receiving aminobisphosphonate treatment for $>3$ years.

\section{Cell culture}

Bone marrow was harvested from the femoral medullary cavity of patients undergoing routine surgery and hBMSCs isolated according to a well established protocol [24]. Purified hBMSCs were maintained at $37{ }^{\circ} \mathrm{C}$, in $5 \% \mathrm{CO}_{2}$ and $98 \%$ humidity in normal growth medium consisting of Dulbecco's modified eagle medium (DMEM-low glucose, with L-Glutamine) (PAA Laboratories GmbH, Pasching, Austria), supplemented with 10\% fetal bovine serum (FBS) (Biowest, Nuaillé, France) and 1\% penicillin (50 units $/ \mathrm{ml}$ ) and streptomycin $(50 \mu \mathrm{g} / \mathrm{ml})$ (PAA Laboratories $\mathrm{GmbH}$, Pasching, Austria). The medium was changed twice a week. Cells were used at passage 4 unless otherwise stated. For all experiments hBMSCs were cultured in 96-, 48- and 6-well culture plates (Sarstedt, Wiener Neudorf, Austria), seeded at densities of $4 \times 10^{3}$ and $5 \times 10^{4}$ cells per well respectively. After $72 \mathrm{~h}$, cells were washed and used for experimental analysis.

\section{Fluorescence activated cell sorting (FACS) analysis of surface markers}

hBMSCs were directly stained with phycoerythrin labeled antibodies (Biozym Scientific GmbH, Oldendorf, Germany) specific for CD90, CD105, CD166, CD34 and CD45. Specificity was confirmed through the use of appropriate phycoerythrin-labeled isotype control antibodies and viability assessed using 7-AAD (1 $\mu \mathrm{g} / \mathrm{ml})$ (Sigma, Vienna, Austria). Flow cytometry was carried out on a FACScan (BD Biosciences, Schwechat, Austria) and staining analyzed using WinMDI2.8 freeware. 
hBMSC differentiation

hBMSCs were induced to undergo either adipogenesis or osteogenesis using well-recognized differentiation protocols previously established in our laboratory [25]. Adipocyte formation was confirmed by positive staining of triglyceride using Oil red O (Sigma-Aldrich, Vienna, Austria). In order to investigate the effects of aminobisphosphonate treatment on hBMSC osteogenesis, osteogenic induction medium (OM) consisting of normal growth medium supplemented with $50 \mu \mathrm{M}$ L-Ascorbic acid 2-phosphate (Sigma-Aldrich), 10mM $\beta$-glycerophosphate (Sigma-Aldrich) and 100nM dexamethasone (Sigma-Aldrich), was supplemented with zoledronate (10 and $100 \mathrm{nM})$ where stated. Mineralization was visualized using Alizarin red and the amount of staining quantified in pooled samples from individual patients using a spectrophotometer following Alizarin red extraction using cetylpyridinium chloride (Sigma-Aldrich, Vienna, Austria). Optical densities were converted to nanomoles (nM) of Alizarin red using a standard curve and normalized to cell number. The mean cell number was determined by automated counting of Hoechst 33258 stained nuclei in at least 9 random fields of view. Images were captured on a Leica DMI 3000 B inverse fluorescence microscope equipped with a DIC 450 C camera and LAS V 4.0 software (all Leica, Microsystems, Wetzlar, Germany). Image processing and nucleus counts were performed with Cell Profiler cell image analysis software version 2.0 (Scientific Volume Imaging, Hilversum, The Netherlands). ALP activity was quantified in cell lysates using pnitrophenylphosphate (pNPP) liquid substrate (Sigma-Aldrich, Buchs, Switzerland) and normalized to total protein content and reaction time. The expression levels of differentiation markers specific to adipogenesis or osteogenesis were measured by quantitative polymerase chain reaction (qRT-PCR) as detailed below.

$q R T-P C R$

Total RNA was purified from hBMSCs using TRI Reagent (Sigma-Aldrich, Vienna, Austria) and treated with TURBO DNase (Life Technologies, Zug, Switzerland). Equal amounts of RNA were combined to 
form the groups Control (BMSCs from untreated patients; $n=5$ ), Control+ZA (BMSCs from untreated patients treated with zoledronate in vitro; $\mathrm{n}=5$ ), ALN (BMSCs from patients treated with alendronate; $\mathrm{n}=3$ ), ALN+ZA (BMSCs from patients treated with alendronate and further treated with zoledronate in vitro; $\mathrm{n}=3)$. RNA $(0.5 \mu \mathrm{g})$ was reverse-transcribed using Superscript II (Life Technologies) as described previously [25]. An equivalent of 10 or $20 \mathrm{ng}$ total RNA was applied as cDNA template in the successive qRT-PCR reactions using the StepOnePlus (Life Technologies). Quantification of mRNA expression was performed with TaqMan Gene Expression Assays (Life Technologies) specific for runt-related transcription factor 2 (RUNX2) (Hs01047976_m1), osteocalcin (OCN) (Hs01587814_g1), secreted phosphoprotein 1/osteopontin (SPP1) (Hs00959010_m1) and fibroblast growth factor (FGF2) (Hs00266645_m1), and values normalized to the housekeeping gene, beta glucuronidase (GUSB) (Hs99999908_m1). All reactions were performed in fast optical 96-well reaction plates (Life Technologies) at $95{ }^{\circ} \mathrm{C}$ for $20 \mathrm{~s}, 40$ cycles of $95{ }^{\circ} \mathrm{C}$ for $1 \mathrm{~s}$ and $60{ }^{\circ} \mathrm{C}$ for $20 \mathrm{sec}$. The mean fold changes from three separate experiments were analyzed using the $2^{-\Delta \Delta \mathrm{CT}}$ method.

\section{SPP1 small interfering RNA (siRNA)}

Specific knock down of SPP1 expression was performed with Silencer Select siRNA oligos, (Ambion, Life Technologies) according to the manufacturer's protocol. Osteoporotic hBMSCs $\left(1 \times 10^{5}\right.$ cells $)$ were transfected with $100 \mathrm{nM} \mathrm{SPP1-specific} \mathrm{(S13376)} \mathrm{or} \mathrm{negative} \mathrm{control} \mathrm{siRNA} \mathrm{(Negative} \mathrm{Control-1)} \mathrm{using}$ the NEON Transfection System (Life Technologies) as previously described [25]. Following transfection, cells were seeded in cell culture plates with fresh growth medium (without antibiotics) and incubated for $24 \mathrm{~h}$ at $37^{\circ} \mathrm{C}, 5 \% \mathrm{CO}_{2}$. Medium was then replaced with either fresh growth medium or differentiation medium and total RNA harvested at day 7 to confirm SPP1 mRNA knockdown. Mineral formation was assessed after 28 days using Alizarin red. 
Western blot analysis of osteopontin

Protein was extracted using CelLytic M (Sigma-Aldrich) containing a protease inhibitor cocktail (SigmaAldrich) and protein amounts determined by BioRad Protein Assay (BioRad, Reinach, Switzerland). Protein samples were boiled for $5 \mathrm{~min}$ in loading buffer (50 mM Tris-HCl, $\mathrm{pH} 6.8,2 \% \mathrm{SDS}, 10 \%$ glycerol, $100 \mathrm{mM}$ DTT, 0.002\% Bromophenol blue) and equal amounts of protein loaded onto 4-15\% Mini-PROTEAN TGX Precast gels (BioRad). Protein was then electroblotted onto PVDF membranes using the Trans-Blot Turbo blotting system (BioRad) and incubated in 5\% skimmed milk, $50 \mathrm{mM}$ Tris$\mathrm{HCl}, \mathrm{pH} 7.6,150 \mathrm{mM} \mathrm{NaCl}, 0.1 \%$ Tween 20 (TBST) for $1 \mathrm{~h}$ at room temperature. Membranes were then incubated overnight at $4^{\circ} \mathrm{C}$ with either rabbit anti-human osteopontin $(1: 1000)$ (Abcam, UK) or mouse anti-human tubulin (1:4000) (Sigma-Aldrich). After washing in TBST three times for 5 min each, membranes were incubated with a HRP-conjugated anti-mouse IgG (1:10’000) (Jackson ImmunoResearch, UK) for $1 \mathrm{~h}$ at room temperature. Following a further washing step, peroxidase activity was detected using SuperSignal West Dura Chemiluminescent Substrate (Thermo Scientific, Lausanne, Switzerland).

\section{Statistical analysis}

All statistical analyses were carried out using SPSS20.0 (SPSS Inc., Chicago, IL). Parametric analysis of data was performed using the two-tailed unpaired Student's $t$-test for comparison of two groups or oneway analysis of variance (ANOVA) followed by Tukey's post-hoc test for multiple group comparisons. In all cases, a $p$-value of $<0.05$ was considered statistically significant, and all data were expressed as mean \pm standard deviation (SD).

\section{Results}

Characterization of hBMSCs isolated from osteoporotic patients

hBMSCs isolated from osteoporotic patients expressed high levels of various markers associated with mesenchymal stem cells, including CD90, CD105, and CD166, and low levels of haematopoietic markers 
CD34 and CD45 (Fig. 1a). Undifferentiated cells displayed typical fibroblast-like appearance (Fig. 1b) and multipotency was also demonstrated in these cell populations through their ability to differentiate into adipocytes (Fig. 1c) and osteoblasts (Fig. 1d).

Effect of zoledronate on the mineral formation by differentiating hBMSCs isolated from osteoporotic patients

hBMSCs from osteoporotic patients were further evaluated for their ability to undergo osteogenic differentiation and subsequent mineralization following their incubation for up to 21 days in osteogenic induction medium (OM) supplemented with or without 10 or $100 \mathrm{nM}$ zoledronate. The degree of mineral formation was visualized and quantified by Alizarin red staining. Treatment with zoledronate consistently enhanced mineral formation over the 3 week treatment period in a concentration dependent manner (Fig. 2a and b). Significant increases in Alizarin red staining at 2 weeks post induction were only observed in cell cultures exposed to the highest concentration of zoledronate tested (100 nM; $p<0.05)$, although a significant effect on mineral formation was observed with both $10(p<0.01)$ or $100 \mathrm{nM}(p<0.01)$ zoledronate by 3 weeks (Fig. $2 \mathrm{a}$ and $\mathrm{b}$ ). The lower concentration of zoledronate (10 $\mathrm{nM}$ ) had no significant effect on cell numbers during the 3 weeks of treatment as compared to cells treated without zoledronate (Fig. 2c). However, cell numbers in cultures treated with the higher concentration of zoledronate $(100 \mathrm{nM})$ were significantly reduced at day $14(p<0.05)$ and at day $21(p<0.01)$ and remained at levels comparable to those observed in uninduced cells (Fig. 2c).

Comparison between osteogenic differentiation of hBMSCs isolated from untreated and alendronatetreated osteoporotic patients

We next isolated hBMSCs from osteoporotic patients undergoing alendronate therapy (ALN) and directly compared their osteogenic potential with hBMSCs from osteoporotic patients without bisphosphonate medication (Control). In all cases, hBMSCs isolated from alendronate-treated patients demonstrated a greater tendency towards osteoblast formation than patients not receiving alendronate treatment as 
confirmed by significant increases in Alizarin red staining at days $14(p<0.01)$ and $21(p<0.01)$ (Fig. 3a and b). In addition, hBMSCs from osteoporotic patients receiving alendronate medication were also induced to undergo osteogenesis for up to 21 days in osteogenic medium supplemented with zoledronate at $10(\mathrm{ALN}+10 \mathrm{nM} \mathrm{ZA})$ or $100 \mathrm{nM}(\mathrm{ALN}+100 \mathrm{nM} \mathrm{ZA})$. In contrast to our initial findings using hBMSCs isolated from non-bisphosphonate treated osteoporotic patients, zoledronate was only capable of enhancing mineralization in cultures of differentiating BMSCs isolated from alendronate-treated patients after 3 weeks of treatment, and only when the highest concentration of zoledronate was used (ALN+100 nM ZA vs. ALN; $p<0.01$ ). However, these increases in mineralization due to zoledronate treatment $(\mathrm{ALN}+100 \mathrm{nM})$ were also associated with significant reductions in cell numbers as compared to control cultures at day $14(p<0.01)$ and day $21(p<0.01)$ (Fig. 3c).

Effect of aminobisphosphonate treatment on ALP activity and osteogenic gene expression in differentiating $h B M S C s$

In order to ascertain whether the stimulatory effects of aminobisphosphonate treatment on hBMSC osteogenic activity were also apparent at the biochemical and molecular level, we analyzed both ALP protein activity and the expression levels of several osteogenic markers in cultures of hBMSCs undergoing osteogenic differentiation. A 2- to 3-fold increase in ALP enzyme activity was calculated for differentiating hBMSCs from each treatment group by day 14 following osteogenic induction (Fig. 4a). However, despite our previous observation that exposure to aminobisphosphonate greatly enhances hBMSC-derived osteoblast mineralization, we were unable to observe any significant increases in ALP enzyme activity in hBMSCs treated with zoledronate (ZA; $100 \mathrm{nM})$ or in hBMSCs isolated from patients treated with alendronate (ALN). The expression levels of $R U N X 2$ did not significantly change during osteogenic differentiation in any of the groups tested (Fig. 4b). Expression levels of FGF2 (Fig. 4c) and OCN (Fig. 4d) were significantly reduced upon osteogenic induction, and were found to be comparable between all treatment groups. In contrast, both zoledronate and alendronate had a marked inhibitory effect on SPP1 gene expression (Fig. 4e). Zoledronate induced a significant reduction in SPP1 expression in 
hBMSCs from untreated patients $($ Control $+Z A)$ at $7(0.4$-fold $\pm 0.09 ; p<0.01)$ and $14(0.4$-fold \pm 0.06 ; $p<0.01)$ days following osteogenic induction. Similarly, SPP1 expression was also significantly reduced in hBMSCs from alendronate-treated patients (ALN) at day $7(0.4$-fold $\pm 0.07 ; p<0.01)$ post osteogenic induction. Zoledronate treatment of these cells $(\mathrm{ALN}+\mathrm{ZA})$ enhanced this effect by inducing a more sustained decrease in SPP1 expression for up to 14 days $(p<0.05)$.

Effect of osteopontin on the mineralization efficiency of differentiating bone-forming cells isolated from osteoporotic patients.

Further studies were performed in order to assess the involvement of osteopontin in determining the mineralizing capabilities of osteoporotic hBMSC-derived osteoblasts. Western blot analysis revealed marked reductions in osteopontin protein levels in differentiating osteoporotic hBMSCs treated with either 10 or $100 \mathrm{nM}$ zoledronate for 10 days as compared to control cultures (Fig. 5a). Loss-of-function studies in differentiating osteoporotic hBMSCs through the use of a siRNA specific for SPPI resulted in a $>140$-fold decrease in SPP1 mRNA expression as compared to cells transfected with a siRNA control oligonucleotide (Fig. 5b). Subsequently, these cells demonstrated a noticeable increase in mineral formation after 28 days of osteogenic induction as compared to cells treated with the siRNA control oligonucleotide (Fig. 5c).

\section{Discussion}

Bisphosphonates are considered as one of the first-line therapies for osteoporosis and are the most commonly prescribed agents to prevent bone loss and subsequent fractures $[26,27]$. Their selective adsorption to mineral surfaces and uptake by resident osteoclasts has given rise to the generally accepted opinion that bisphosphonates exert their anti-resorptive effects primarily through modification of hydroxyapatite crystal dissolution and osteoclast activity [28]. Indeed, numerous studies have confirmed that bisphosphonates can directly influence osteoclast resorptive activity through alterations in osteoclast 
formation and viability [29-32]. Moreover, the cellular and biochemical mechanisms through which these effects are brought about have since been elucidated, thus providing us with an even deeper understanding of how bisphosphonates may function to prevent bone loss [33].

It has been known for some time that bisphosphonates have the potential to prevent apoptosis in boneforming osteoblasts and osteocytes in vitro [34,35], although information relating to their ability to directly influence bone formation through stimulation of such cells remains limited [36, 37]. It is becoming increasingly evident however, that bisphosphonates may have an indirect effect on bone quality through their potential to enhance osteogenesis in differentiating BMSCs. Although thought to represent only 0.001 to $0.01 \%$ of the total cell population found in bone marrow [38], BMSCs play a crucial role in maintaining normal bone homeostasis through their capacity for osteoblastic differentiation [39]. The influence of bisphosphonates on BMSC-derived osteoblast formation was originally described by Giuliani et al. [18], where cultures of bone marrow cells treated with low doses of either etidronate or alendronate resulted in increased numbers of colony forming units for osteoblasts (CF-OBs). Moreover, bone marrow cells isolated from bisphosphonate-treated mice also demonstrated an increased potential for CF-OB formation, thus suggesting that the stimulatory effects of bisphosphonates on osteoblastogenesis were also evident in vivo. Several more reports have since confirmed these initial findings using different bisphosphonates and alternative stem cell sources [19-22]. However, up until now, no studies have sought to determine the effects of bisphosphonates on hBMSCs isolated from osteoporotic patients and to evaluate their potential osteoanabolic influence on these cells.

In the current report, we successfully isolated and characterized hBMSCs from aged female osteoporotic patients. We have confirmed our hypothesis that in vitro treatment of these hBMSC populations with zoledronate during osteogenic differentiation could greatly enhance their potential for osteoblastogenesis as demonstrated by a significant increase in mineral deposition. Moreover, we could also demonstrate that hBMSCs isolated from osteoporotic patients receiving treatment with alendronate were also subject to enhanced osteoblastogenesis under normal osteogenic culture conditions. The potential for alendronate to 
enhance osteoblast formation in vivo may represent an additional mechanism by which bone mineral density (BMD) is increased in osteoporotic patients receiving aminobisphosphonate therapy [40]. Furthermore, the fact that we were able to visualize enhanced mineral formation in osteoblasts derived from these hBMSCs in the absence of further in vitro aminobisphosphonate stimulation, suggests that these cells have the potential to retain their enhanced osteogenic capacity, a trait that may also exist in vivo during extended periods of bisphosphonate discontinuation. If confirmed, this theory may be of clinical importance when considering replacement therapies during time off from bisphosphonate treatment [41]. Moreover, such findings may also be of significant relevance when considering the long term effects of aminobisphosphonate treatment. Numerous reports now exist linking atypical femoral fractures in osteoporotic patients with long-term bisphosphonate treatment, possibly through increases in bone mineralization and brittleness due to decreases in bone turnover [42-45]. Our discovery that longterm bisphosphonate therapy enhances hBMSC-derived osteoblast mineralization, may therefore constitute an additional mechanism through which bisphosphonates influence bone mineralization and subsequent susceptibility to atypical femoral fractures, independent of their anti-osteoclastic effects.

Although increases in osteoporotic hBMSC-derived mineral formation induced by high concentrations of zoledronate $(100 \mathrm{nM})$ could partly be explained by reductions in cell numbers, this could not account for the enhanced levels of mineralization induced by either low zoledronate concentrations $(10 \mathrm{nM})$ or by hBMSCs isolated from alendronate-treated osteoporotic patients. The mechanism of action of aminobisphosphonates on BMSC-derived mineral formation remains largely undetermined, although previous reports have alluded to the fact that aminobisphosphonates may mediate their effects through the regulation of various proteins and genes specific to osteogenesis. The activities of alkaline phosphatase (ALP) are considered to be an essential prerequisite for the successful initiation of mineralization [46]. As such, ALP activity levels are generally used as a measure of osteogenesis in differentiating MSC cultures in vitro. Despite the fact that we could demonstrate marked increases in mineral formation in hBMSC cultures previously exposed to either zoledronate or alendronate, ALP activity in these cells remained 
unaffected. These findings are in line with previous studies in which the effects of zoledronate and alendronate on hBMSC osteogenesis were evaluated [19, 47]. However, additional reports also exist describing the stimulatory effects of both zoledronate and alendronate on ALP activity in differentiating hBMSCs $[20,21]$. As such, it remains unclear as to the involvement of ALP in regulating the stimulatory effects of aminobisphosphonate treatment on hBMSC osteogenesis and subsequent mineral formation. At least in the hBMSCs of the osteoporotic individuals used in the current study, ALP activity did not appear to represent a critical factor in the mineral-promoting actions of either zoledronate or alendronate.

At the genetic level, increases in the expression of $F G F 2, R U N X 2, B S P 2$ and $B M P-2$ mRNA have been observed in hBMSCs treated with alendronate or zoledronate [19, 48]. Similarly, osteocalcin and RUNX2 protein levels were also found to be elevated in hBMSCs undergoing osteogenic differentiation in response to alendronate treatment [20]. In contrast, a more recent study by Ebert et al (2009) [21] demonstrated that the stimulatory effects of zoledronate on hBMSC mineralization were associated with a downregulation in the expression levels of osteogenic markers OCN, SPP1 and COL1A2. In the present report, $R U N X 2$ expression levels were unaltered during osteogenesis and remained at comparable levels in all treatment groups. The expression levels of both $F G F 2$ and $O C N$ were significantly downregulated upon osteogenic induction in either the presence or absence of aminobisphosphonate. As such, we do not consider the stimulatory effects of aminobisphosphonate treatment to be dependent on alterations in these osteogenic markers. However, aminobisphosphonate treatment did induce a significant downregulation in osteopontin at both mRNA and protein levels in hBMSCs undergoing osteogenic differentiation as compared to untreated control cells. Osteopontin is generally considered to be a negative regulator of osteogenic differentiation as demonstrated by the fact that cortical bone mineralization is significantly enhanced in $\mathrm{Sppl}^{-/}$mice [49]. Furthermore, deletion of the Sppl gene from hypomineralized mice deficient in the tissue-specific ALP gene, $A k p 2$, partially restored a normal bone phenotype in these mice [50]. This, along with its ability to inhibit mineral formation in vitro [51], would suggest that downregulation of SPP1 expression in osteoblastic cultures by aminobisphosphonates may represent a 
plausible means through which they enhance mineral deposition both in vitro and possibly even in vivo. This theory is further supported by the results from our loss-of-function studies in which SPP1 gene knockdown enhanced mineral formation in osteoporotic hBMSCs undergoing osteogenic differentiation. A proposed limitation of this study is that hBMSCs were isolated from female osteoporotic patients only, and thus we cannot exclude possible gender-related effects with regards to the differentiation potential of the isolated hBMSCs. Despite females representing the majority of osteoporotic (hip) fracture patients, further investigations into the osteogenic potential of BMSCs from male osteoporotic patients may therefore be warranted. Furthermore, our study is somewhat underpowered due to the limited number of aged ( $>65$ years old) osteoporotic patients that could be recruited for donation of hBMSCs. This was especially evident in the case of acquiring samples from osteoporotic patients receiving aminobisphosphonate medication. Future studies using larger numbers of patients, in combination with more diverse treatment groups, would certainly help clarify the overall impact of bisphosphonate treatment on hBMSC osteogenesis and mineralization of differentiating bone-forming cells isolated from osteoporotic patients.

In summary, we demonstrate here that exposure of osteoporotic hBMSCs to aminobisphosphonates greatly enhances their capacity for osteoblast-mediated mineral formation, and may thus represent an alternative means by which bisphosphonates increase BMD in treated patients. Furthermore, downregulation of osteopontin production is implicated as a possible mechanism of action. It is expected that further investigations into how bisphosphonates influence hBMSC function will better our understanding of how these potent synthetic compounds act to mediate bone quality and turnover in osteoporotic patients, and that these insights may ultimately lead to the generation of new and novel approaches to treat age-related osteoporotic bone loss. 


\title{
Conflict of interest
}

All authors have no conflicts of interest and have nothing to disclose.

\begin{abstract}
Acknowledgements
This study was supported by funding received from the Mäxi/CABMM Start-up grant, AOTrauma, Novartis Foundation, formerly Ciba-Geigy-Jubilee-Foundation, and Uniscientia. The authors would like to express their gratitude to Novartis (Vienna, Austria) for supplying the zoledronate used in this study.
\end{abstract}

\section{References}

1. Zaidi M (2007) Skeletal remodeling in health and disease. Nat Med 13:791-801

2. Raggatt LJ, Partridge NC (2010) Cellular and molecular mechanisms of bone remodeling. J Biol Chem 285:25103-8

3. Rodríguez JP, Garat S, Gajardo H, Pino AM, Seitz G (1999) Abnormal osteogenesis in osteoporotic patients is reflected by altered mesenchymal stem cells dynamics. J Cell Biochem 75:414-23

4. Rodríguez JP, Montecinos L, Rios S, Reyes P, Martinez J (2000) Mesenchymal stem cells from osteoporotic patients produce a type I collagen-deficient extracellular matrix favoring adipogenic differentiation. J Cell Biochem 79:557-65

5. Hess R, Pino AM, Ríos S, Fernández M, Rodríguez JP (2005) High affinity leptin receptors are present in human mesenchymal stem cells (MSCs) derived from control and osteoporotic donors. J Cell Biochem 94:50-7

6. Perrini S, Natalicchio A, Laviola L, et al (2008) Abnormalities of Insulin-Like Growth Factor-I Signaling and Impaired Cell Proliferation in Osteoblasts from Subjects with Osteoporosis. Endocrinology 149:1302-13

7. Zhang ZM, Jiang LS, Jiang SD, Dai LY (2009) Osteogenic potential and responsiveness to leptin of mesenchymal stem cells between postmenopausal women with osteoarthritis and osteoporosis. J Orthop Res 27:1067-73 
8. Dalle Carbonare L, Valenti MT, Zanatta M, Donatelli L, Lo Cascio V (2009) Circulating mesenchymal stem cells with abnormal osteogenic differentiation in patients with osteoporosis. Arthritis Rheum 60:3356-65

9. Egermann M, Heil P, Tami A, Ito K, Janicki P, Von Rechenberg B, Hofstetter W, Richards PJ (2010) Influence of defective bone marrow osteogenesis on fracture repair in an experimental model of senile osteoporosis. J Orthop Res 28:798-804

10. Jilka RL, Weinstein RS, Takahashi K, Parfitt AM, Manolagas SC (1996) Linkage of decreased bone mass with impaired osteoblastogenesis in a murine model of accelerated senescence. J Clin Invest $97: 1732-1740$

11. Silva MJ, Brodt MD, Ko M, Abu-Amer Y (2005) Impaired Marrow osteogenesis is associated with reduced endocortical bone formation but does not impair periosteal bone formation in long bones of SAMP6 mice. J Bone Miner Res 20:419-427

12. Kuro-o M, Matsumura Y, Aizawa H, et al (1997) Mutation of the mouse klotho gene leads to a syndrome resembling aging. Nature 390:45-51

13. Kawaguchi H, Manabe N, Miyaura C, Chikuda H, Nakamura K, Kuro-o M (1999) Independent impairment of osteoblast and osteoclast differentiation in klotho mouse exhibiting low- turnover osteopenia. J Clin Invest 104:229-237

14. Bonyadi M, Waldman SD, Liu D, Aubin JE, Grynpas MD, Stanford WL (2003) Mesenchymal progenitor self-renewal deficiency leads to age-dependent osteoporosis in Sca-1/Ly-6A null mice. Proc Natl Acad Sci U S A 100:5840-5845

15. Choi Y, Wright AC, Johnson FB (2008) Defects in telomere maintenance molecules impair osteoblast differentiation and promote osteoporosis. Aging Cell 7:23-31

16. Rachner TD, Khosla S, Hofbauer LC (2011) Osteoporosis: now and the future. Lancet 377:1276-87

17. Li B, Ling Chau JF, Wang X, Leong WF (2011) Bisphosphonates, specific inhibitors of osteoclast function and a class of drugs for osteoporosis therapy. J Cell Biochem 112:1229-42 
18. Giuliani N, Pedrazzoni M, Negri G, Passeri G, Impicciatore M, Girasole G (1998) Bisphosphonates stimulate formation of osteoblast precursors and mineralized nodules in murine and human bone marrow cultures in vitro and promote early osteoblastogenesis in young and aged mice in vivo. Bone 22:455-61

19. von Knoch F, Jaquiery C, Kowalsky M, et al (2005) Effects of bisphosphonates on proliferation and osteoblast differentiation of human bone marrow stromal cells. Biomaterials 26:6941-9

20. Duque G, Rivas D (2007). Alendronate has an anabolic effect on bone through the differentiation of mesenchymal stem cells. J Bone Miner Res 22:1603-11

21. Ebert R, Zeck S, Krug R, et al (2009) Pulse treatment with zoledronic acid causes sustained commitment of bone marrow derived mesenchymal stem cells for osteogenic differentiation. Bone $44: 858-64$

22. Wang C-Z, Chen S-M, Chen C-H, et al (2010) The effect of the local delivery of alendronate on human adipose-derived stem cell-based bone regeneration. Biomaterials 31:8674-83

23. Benisch P, Schilling T, Klein-Hitpass L, et al (2012) The transcriptional profile of mesenchymal stem cell populations in primary osteoporosis is distinct and shows overexpression of osteogenic inhibitors. PLoS One 7:e45142

24. Wolfe M, Pochampally R, Swaney W, Reger RL (2008) Isolation and culture of bone marrowderived human multipotent stromal cells (hMSCs). In: Prockop FJ, Phinney DC, Bunnell BA (eds) Methods in Molecular Biology. Humana Press, pp 449:3-25.

25. Tiaden AN, Breiden M, Mirsaidi A, Weber FA, Bahrenberg G, Glanz S, Cinelli P, Ehrmann M, Richards PJ (2012) Human Serine Protease HTRA1 Positively Regulates Osteogenesis of Human Bone Marrow-derived Mesenchymal Stem Cells and Mineralization of Differentiating Bone-forming Cells Through the Modulation of Extracellular Matrix Protein. Stem Cells 30:2271-82

26. Boonen S, Laan RF, Barton IP, Watts NB (2005) Effect of osteoporosis treatments on risk of nonvertebral fractures: review and meta-analysis of intention-to-treat studies. Osteoporos Int 16:1291-8 
27. Eastell R, Walsh JS, Watts NB, Siris E (2011) Bisphosphonates for postmenopausal osteoporosis. Bone 49:82-88

28. Russell RGG (2011) Bisphosphonates: The first 40 years. Bone 49:2-19

29. Flanagan AM, Chambers TJ (1989) Dichloromethylenebisphosphonate (Cl2MBP) inhibits bone resorption through injury to osteoclasts that resorb Cl2MBP-coated bone. Bone Miner 6:33-43

30. Hughes DE, Wright KR, Uy HL, Sasaki A, Yoneda T, Roodman GD, Mundy GR, Boyce BF (1995) Bisphosphonates promote apoptosis in murine osteoclasts in vitro and in vivo. J Bone Miner Res $10: 1478-87$

31. Breuil V, Cosman F, Stein L, Horbert W, Nieves J, Shen V, Lindsay R, Dempster DW (1998) Human osteoclast formation and activity in vitro: effects of alendronate. J Bone Miner Res 13:17219

32. Hughes DE, McDonald BR, Russell RGG, Gowen M (1989) Inhibition of osteoclast-like cell formation by bisphosphonates in long-term culture of human bone marrow. J Clin Invest 83:1930-5

33. Rogers MJ, Crockett JC, Coxon FP, Mönkkönen J (2011) Biochemical and molecular mechanisms of action of bisphosphonates. Bone 49:34-41

34. Plotkin LI, Bivi N, Bellido T (2011) A bisphosphonate that does not affect osteoclasts prevents osteoblast and osteocyte apoptosis and the loss of bone strength induced by glucocorticoids in mice. Bone 49:122-7

35. Plotkin LI, Weinstein RS, Parfitt AM, Roberson PK, Manolagas SC, Bellido T (1999) Prevention of osteocyte and osteoblast apoptosis by bisphosphonates and calcitonin. J Clin Invest 104:1363-74

36. Reinholz GG, Getz B, Pederson L, Sanders ES, Subramaniam M, Ingle JN, Spelsberg TC (2000) Bisphosphonates directly regulate cell proliferation, differentiation, and gene expression in human osteoblasts. Cancer Res 60:6001-7

37. Im G-I, Qureshi SA, Kenney J, Rubash HE, Shanbhag AS (2004) Osteoblast proliferation and maturation by bisphosphonates. Biomaterials 25:4105-15 
38. Pittenger MF, Mackay AM, Beck SC, Jaiswal RK, Douglas R, Mosca JD, Moorman MA, Simonetti DW, Craig S, Marshak DR (1999) Multilineage potential of adult human mesenchymal stem cells. Science 284:143-7

39. Seeman E, Delmas PD (2006) Bone quality--the material and structural basis of bone strength and fragility. N Engl J Med 354:2250-61

40. Bone HG, Hosking D, Devogelaer JP, Tucci JR, Emkey RD, Tonino RP, Rodriguez-Portales JA, Downs RW, Gupta J, Santora AC, Liberman UA (2004) Alendronate Phase III Osteoporosis Treatment Study Group. Ten years' experience with alendronate for osteoporosis in postmenopausal women. N Engl J Med 350:1189-99

41. Diab DL, Watts NB (2012) Bisphosphonates in the treatment of osteoporosis. Endocrinol Metab Clin North Am 41:487-506

42. Thompson RN, Phillips JR, McCauley SH, Elliott JR, Moran CG (2012) Atypical femoral fractures and bisphosphonate treatment: experience in two large United Kingdom teaching hospitals. J Bone Joint Surg Br 94:385-90

43. Odvina CV, Levy S, Rao S, Zerwekh JE, Rao DS (2010) Unusual mid-shaft fractures during longterm bisphosphonate therapy. Clin Endocrinol (Oxf) 72:161-8

44. Shane E, Burr D, Ebeling PR, et al (2010) merican Society for Bone and Mineral Research. Atypical subtrochanteric and diaphyseal femoral fractures: report of a task force of the American Society for Bone and Mineral Research. J Bone Miner Res 25:2267-94

45. Kwek EB, Goh SK, Koh JS, Png MA, Howe TS (2008) An emerging pattern of subtrochanteric stress fractures: a long-term complication of alendronate therapy? Injury 39:224-31

46. Golub EE, Boesze-Battaglia K (2007) The role of alkaline phosphatase in mineralization. Curr Opin Orthop 18:444-448

47. Kellinsalmi M, Mönkkönen H, Mönkkönen J, Leskelä HV, Parikka V, Hämäläinen M, Lehenkari P (2005) In vitro comparison of clodronate, pamidronate and zoledronic acid effects on rat osteoclasts and human stem cell-derived osteoblasts. Basic Clin Pharmacol Toxicol 97:382-91 
48. Giuliani N, Girasole G, Pedrazzoni M, Passeri G, Gatti C, Passeri M (1995) Alendronate stimulates b-FGF production and mineralized nodule formation in human osteoblastic cells and osteoblastogenesis in human bone marrow cultures. J Bone Miner Res 10:S171

49. Boskey AL, Spevak L, Paschalis E, Doty SB, McKee MD (2002) Osteopontin deficiency increases mineral content and mineral crystallinity in mouse bone. Calcif Tissue Int 71:145-54

50. Harmey D, Johnson KA, Zelken J, Camacho NP, Hoylaerts MF, Noda M, Terkeltaub R, Millán JL (2006) Elevated skeletal osteopontin levels contribute to the hypophosphatasia phenotype in Akp2(/-) mice. J Bone Miner Res 21:1377-86

51. Addison WN, Azari F, Sørensen ES, Kaartinen MT, McKee MD (2007) Pyrophosphate inhibits mineralization of osteoblast cultures by binding to mineral, up-regulating osteopontin, and inhibiting alkaline phosphatase activity. J Biol Chem 282:15872-83 
Table 1. Details of patients from which BMSCs were isolated.

\begin{tabular}{|c|c|c|c|c|c|c|c|c|c|}
\hline $\begin{array}{l}\text { Patient } \\
\text { (sex) }\end{array}$ & $\begin{array}{l}\text { Age } \\
(\mathrm{yrs})\end{array}$ & $\begin{array}{l}\text { Mobility } \\
\text { score }^{a}\end{array}$ & $\begin{array}{l}\mathrm{T} \text {-score } \\
\text { (femoral } \\
\text { neck) }\end{array}$ & $\begin{array}{l}\text { T-score } \\
\text { (total } \\
\text { hip) } \\
\end{array}$ & $\begin{array}{l}\text { T-score } \\
\text { (lumbar } \\
\text { spine) }\end{array}$ & $\overline{\mathrm{BMI}}$ & $\begin{array}{l}\mathrm{P} 1 \mathrm{NP}^{c} \\
(\mu \mathrm{g} / \mathrm{l})\end{array}$ & $\begin{array}{l}\beta-\mathrm{CTx}^{c} \\
(\mathrm{ng} / \mathrm{l})\end{array}$ & $\begin{array}{l}\text { Anti-osteoporotic } \\
\text { therapy used }\end{array}$ \\
\hline $\bar{F}$ & 84 & 2 & -2.9 & \begin{tabular}{|c|}
-3.3 \\
\end{tabular} & -3 & 18.9 & 47 & 932 & - \\
\hline $\mathrm{F}$ & 92 & 4 & -2.9 & -3.4 & -4.6 & 20.2 & 61 & 1194 & - \\
\hline $\mathrm{F}$ & 89 & 9 & -2.5 & -1.8 & -1 & 23.5 & 64 & 864 & - \\
\hline $\mathrm{F}$ & 95 & 6 & -2.7 & -2.9 & -1.9 & 22.9 & 37 & 345 & - \\
\hline $\mathrm{F}$ & 84 & 4 & n.d. & n.d. & -5.1 & 19.5 & 47 & 376 & - \\
\hline $\mathrm{F}$ & 90 & 4 & -3.5 & -3.6 & -5 & 21.2 & 108 & 1033 & $\mathrm{Ca} /$ Vit.D \\
\hline $\mathrm{F}$ & 85 & 9 & -2.6 & -2.4 & -2.9 & 25.0 & 77 & 490 & \\
\hline $\mathrm{F}$ & 79 & 4 & n.d. & n.d. & -4.2 & 20.8 & 60 & 71 & ${ }^{d}$ Fosamax/Ca/Vit.D \\
\hline $\mathrm{F}$ & 82 & 9 & -3 & -2.6 & -2.4 & 23.2 & 32 & 284 & ${ }^{d}$ Fosamax/Ca/Vit.D \\
\hline $\mathrm{F}$ & 85 & 9 & -2.4 & -2.4 & -3 & 24.0 & 24 & 261 & ${ }^{d}$ Fosamax/Ca/Vit.D \\
\hline
\end{tabular}

${ }^{a}$ Parker mobility score ranging from 0 (no walking ability at all) to 9 (fully independent).

${ }^{b}$ Determined by DXA analysis where a T-score of -2.5 standard deviations (SD) or less was considered osteoporotic.

${ }^{c}$ Bone turnover markers, PINP, Serum procollagen type I N propeptide (bone formation marker); $\beta$-CTx, Serum carboxy-terminal cross-linking telopeptide of type I collagen (bone resorption marker).

${ }^{d}$ Patients received 70mg of Fosamax (alendronate) once weekly and duration of treatment ranged from 412 years.

$F$, female; $B M I$, body mass index; Ca/Vit.D, calcium/vitamin $\mathrm{D} ;$ n.d., not determined due to contralateral implant following previous hip fracture surgery. 
Fig. 1 Characterization of hBMSCs isolated from osteoporotic patients. a Expression of surface antigens by hBMSCs isolated from aged female osteoporotic patients $(n=3)$. The expression of surface markers associated with mesenchymal cells (CD90, CD105 and CD166) and hematopoietic cells (CD34 and CD45) was assessed by fluorescence activated cell sorting (FACS). b Phase contrast image of cultured hBMSCs grown under non-differentiating conditions. c Cells differentiated towards adipocytes and stained with Oil red $\mathrm{O}$ at day 14. d Cells differentiated towards osteoblasts and stained with Alizarin red at day 21. Images are representative of at least three separate experiments

Fig. 2 Effect of zoledronate on the mineral formation by differentiating hBMSCs isolated from osteoporotic patients. hBMSCs isolated from osteoporotic patients $(n=7)$ were induced to undergo osteogenic differentiation for 14 and 21 days in osteogenic medium (OM) with or without zoledronate (10 and $100 \mathrm{nM}$ ) and mineral formation determined by Alizarin red staining. a Representative images of hBMSC cultures isolated from four separate patients stained with Alizarin red at 21 days after osteogenic induction. b Quantitative determination of Alizarin red staining normalized to cell number. c Mean cell number as determined from at least 9 random fields of view. Data is expressed as mean \pm SD. $* p<0.05$, ** $p<0.01$ in comparison to osteogenic medium without ZA (OM) as determined by ANOVA. All experiments were performed in triplicate.

Fig. 3 Comparison between osteogenic differentiation of hBMSCs isolated from untreated and alendronate-treated osteoporotic patients. hBMSCs isolated from osteoporotic patients receiving alendronate treatment $(\mathrm{ALN})(\mathrm{n}=3)$ were induced to undergo osteogenic differentiation for 14 and 21 days with or without zoledronate (ZA) (10 and $100 \mathrm{nM})$. Mineral formation was assessed by Alizarin red staining and compared to hBMSCs isolated from osteoporotic patients of comparable age and T-score not receiving bisphosphonate treatment (Control) $(n=3)$ cultured at the same time under identical conditions. a Representative images of Alizarin red stained hBMSC cultures from each set of patients used. b 
Quantitative determination of Alizarin red staining normalized to cell number. c Mean cell number as determined from at least 9 random fields of view. Data is expressed as mean \pm SD. $* p<0.05, * * p<0.01$ in comparison to control as determined by ANOVA. All experiments were performed in triplicate.

Fig. 4 Effect of aminobisphosphonate treatment on ALP activity and osteogenic gene expression in differentiating hBMSCs. hBMSCs isolated from alendronate treated (ALN; $n=3$ ) or untreated (Control; $n$ =5-6) osteoporotic patients were induced to undergo osteogenic differentiation for up to 21 days with or without zoledronate (ZA) (100 nM). a Cell lysates were harvested at 0, 7, 14 and 21 days following osteogenic induction and ALP activity determined using an ALP activity assay. Expression levels of RUNX2 (b), FGF2 (c), OCN (d), and SPP1 (e) were determined in hBMSCs at days 0, 7 and 14 following osteogenic induction using qRT-PCR and values expressed as fold change in mRNA expression relative to either control hBMSC at day 0 (Control, Control+ZA) or alendronate-treated hBMSCs at day 0 (ALN, $\mathrm{ALN}+\mathrm{ZA})($ value $=1)$. In all cases, experiments were performed in triplicate and data expressed as mean $\pm \mathrm{SD} .{ }^{*} p<0.05,{ }^{* *} p<0.01$ as determined by ANOVA whereby Control and Control+ZA were compared to Control at day 0 , and ALN and ALN+ZA were compared to ALN at day 0 .

Fig. 5 Effect of osteopontin on the mineralization efficiency of differentiating bone-forming cells isolated from osteoporotic patients. a Equal amounts of protein extracts from hBMSCs incubated for 10 days in growth medium (lane 1) or in osteogenic medium alone (lane 2) or supplemented with $10 \mathrm{nM}$ (lane 3) or $100 \mathrm{nM}$ (lane 4) ZA were loaded onto a SDS-PAGE gel and immunoblotting performed using an antibody specific for osteopontin. Levels of tubulin were used as a loading control. b qRT-PCR analysis of SPP1 gene expression in differentiating osteoporotic hBMSCs at day 7 following transfection with either control siRNA (siRNA (C)) or SPP1 siRNA (siRNA (SPP1)). Data was normalized to GUSB and expressed as fold change $(\log 10)$ as compared to cells transfected with siRNA (C) using the $2^{-\Delta \Delta C T}$ method. Experiments were performed in triplicate and data expressed as mean \pm SD. ${ }^{*} p<0.01$, in 
comparison to siRNA (C) as determined by Student's $t$-test. c Representative images of Alizarin red stained osteoporotic hBMSCs transfected with control siRNA (siRNA (C)) or SPPI siRNA (siRNA (SPP1)) at day 28 post-osteogenic induction.

9

10

11

12

13

14

15

16

17

18

19

20

21

22

23

24

25

26

27

28

29

30

31

32

33

34

35

36

37

38

39

40

41

42

43

44

45

46

47

48

49

50

51

52

53

54

55

56

57

58

59

60

http://mc.manuscriptcentral.com/oi 
A
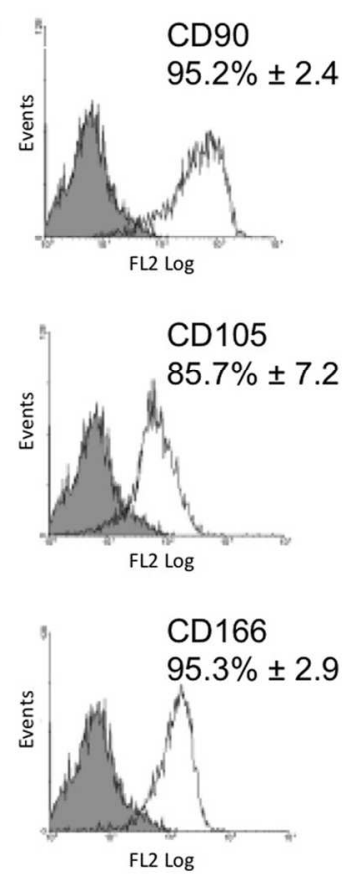
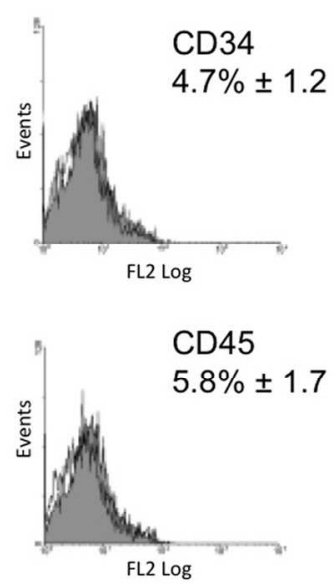

B

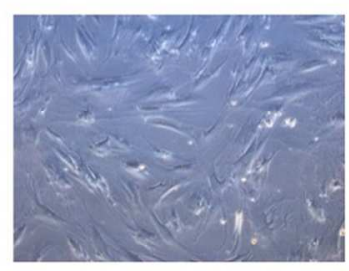

C

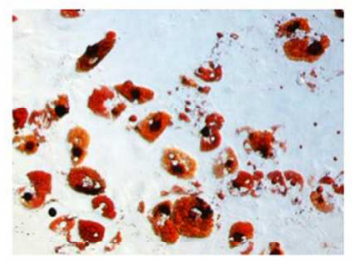

D

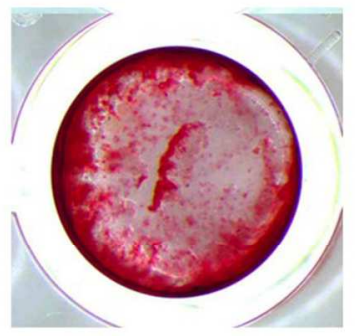

Fig. 1 Characterization of hBMSCs isolated from osteoporotic patients. a Expression of surface antigens by hBMSCs isolated from senile osteoporotic patients $(n=3)$. The expression of surface markers associated with mesenchymal cells (CD90, CD105 and CD166) and hematopoietic cells (CD34 and CD45) was assessed by fluorescence activated cell sorting (FACS). b Phase contrast image of cultured hBMSCs grown under nondifferentiating conditions. c Cells differentiated towards adipogenesis and stained with Oil red $\mathrm{O}$ at day 14. $\mathrm{d}$ Cells differentiated towards osteogenesis and stained with Alizarin red at day 21. Images are representative of at least three separate experiments $72 \times 51 \mathrm{~mm}(600 \times 600 \mathrm{DPI})$ 


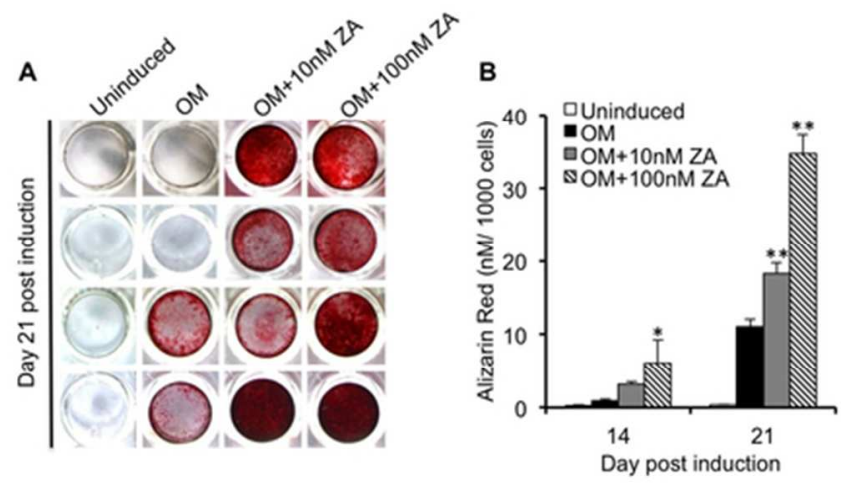

C

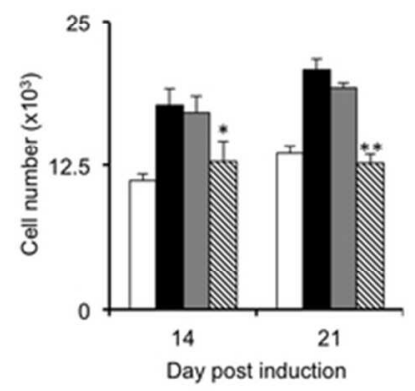

Fig. 2 Effect of zoledronate on the mineral formation by differentiating hBMSCs isolated from osteoporotic patients. hBMSCs isolated from senile osteoporotic patients $(n=7)$ were induced to undergo osteogenic differentiation for 14 and 21 days in osteogenic medium (OM) with or without zoledronate (10 and $100 \mathrm{nM}$ ) and mineral formation determined by Alizarin red staining. a Representative images of hBMSC cultures isolated from four separate patients stained with Alizarin red at 21 days after osteogenic induction. $\mathbf{b}$ Quantitative determination of Alizarin red staining normalized to cell number. c Mean cell number as determined from at least 9 random fields of view. Data is expressed as mean \pm SD. $* p<0.05, * * p<0.01$ in comparison to osteogenic medium without ZA (OM) as determined by ANOVA. All experiments were performed in triplicate. $56 \times 21 \mathrm{~mm}(300 \times 300$ DPI $)$ 
B

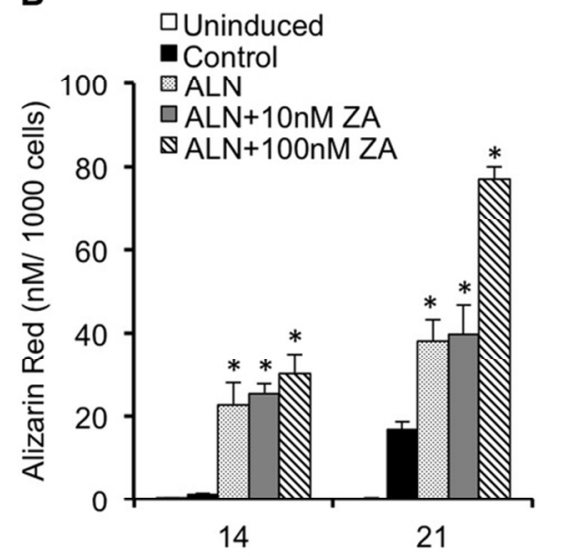

Day post induction

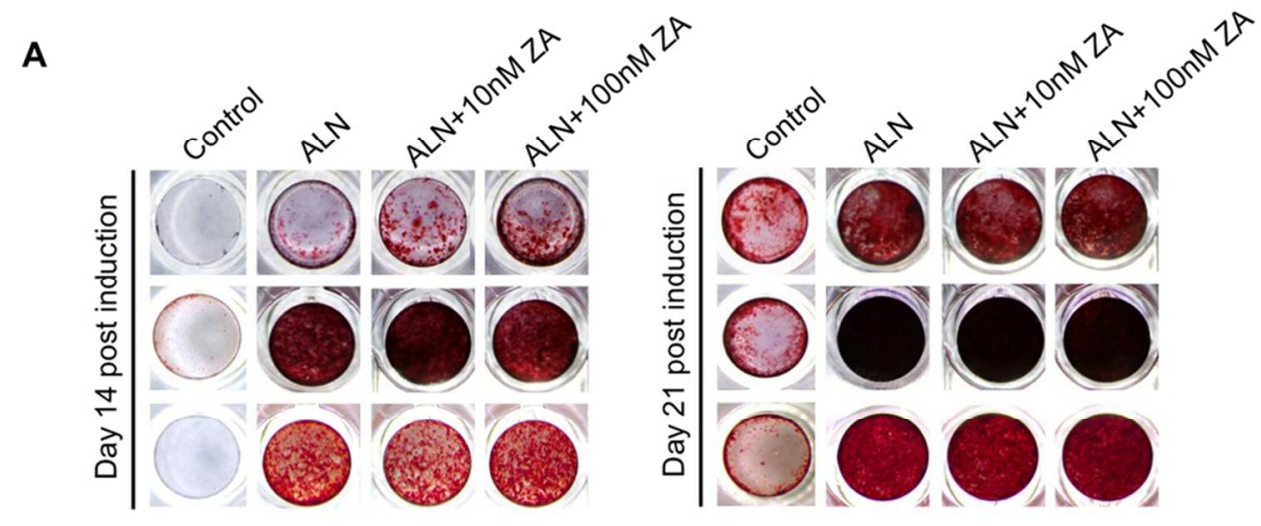

C

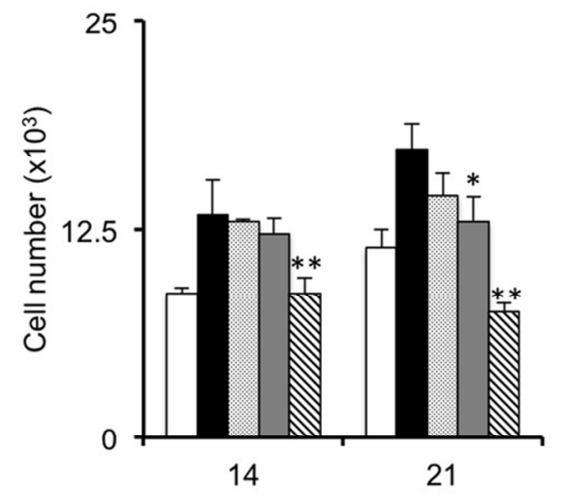

Day post induction

Fig. 3 Comparison between osteogenic differentiation of hBMSCs isolated from untreated and alendronatetreated osteoporotic patients. hBMSCs isolated from senile osteoporotic patients receiving alendronate treatment $(A L N)(n=3)$ were induced to undergo osteogenic differentiation for 14 and 21 days with or without zoledronate (ZA) (10 and $100 \mathrm{nM}$ ). Mineral formation was assessed by Alizarin red staining and compared to hBMSCs isolated from osteoporotic patients of comparable age and T-score not receiving bisphosphonate treatment (Control) $(n=3)$ cultured at the same time under identical conditions. a Representative images of Alizarin red stained hBMSC cultures from each set of patients used. b Quantitative determination of Alizarin red staining normalized to cell number. c Mean cell number as determined from at least 9 random fields of view. Data is expressed as mean \pm SD. $* p<0.05, * * p<0.01$ in comparison to control as determined by ANOVA. All experiments were performed in triplicate. $96 \times 92 \mathrm{~mm}(300 \times 300 \mathrm{DPI})$ 

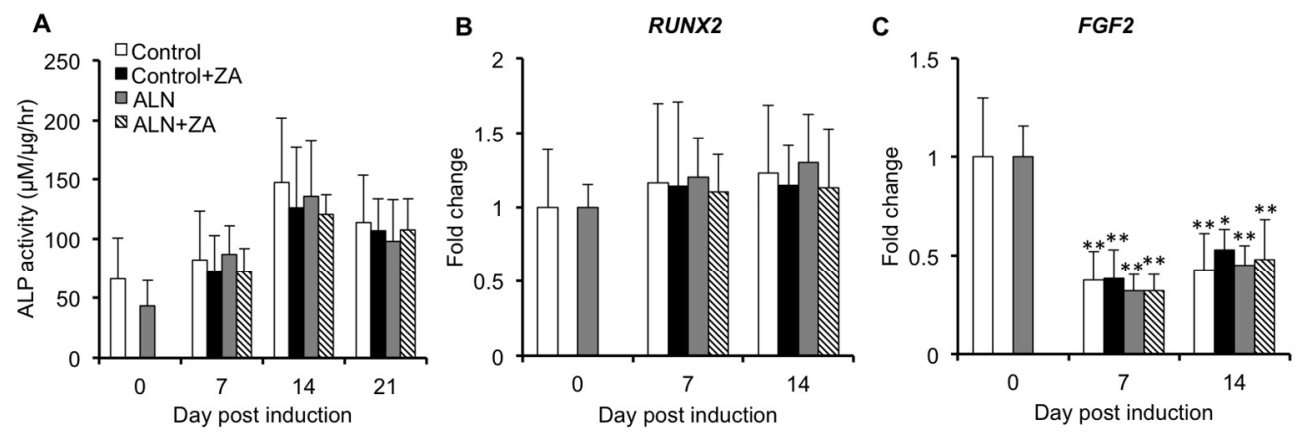

D

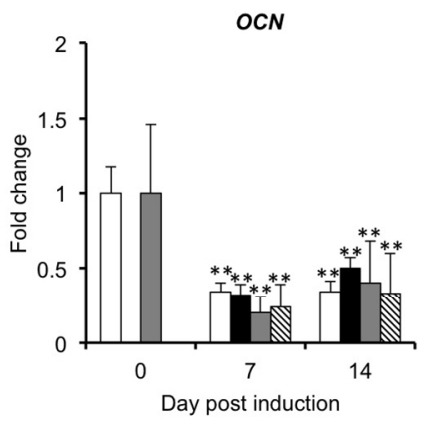

E

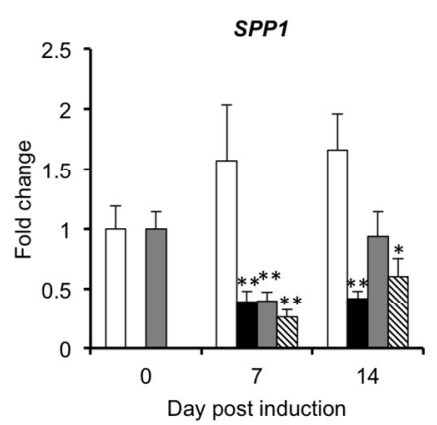

Fig. 4 Effect of aminobisphosphonate treatment on ALP activity and osteogenic gene expression in differentiating hBMSCs. hBMSCs isolated from alendronate treated $(\mathrm{ALN} ; \mathrm{n}=3$ ) or untreated (Control; $\mathrm{n}=$ 5-6) senile osteoporotic patients were induced to undergo osteogenic differentiation for up to 21 days with or without zoledronate (ZA) $(100 \mathrm{nM})$. a Cell lysates were harvested at $0,7,14$ and 21 days following osteogenic induction and ALP activity determined using an ALP activity assay. Expression levels of RUNX2 (b), FGF2 (c), OCN (d) and SPP1 (e) were determined in hBMSCs at days 0, 7 and 14 following osteogenic induction using qRT-PCR and values expressed as fold change in mRNA expression relative to either control hBMSCs at day 0 (Control, Control+ZA) or alendronate-treated hBMSCs at day $0(A L N, A L N+Z A)($ value $=$ 1 ). In all cases, experiments were performed in triplicate and data expressed as mean \pm SD. $* p<0.05$, ** $p<0.01$ as determined by ANOVA whereby Control and Control+ZA were compared to Control at day 0 , and ALN and ALN+ZA were compared to ALN at day 0. $561 \times 401 \mathrm{~mm}(72 \times 72 \mathrm{DPI})$ 
A
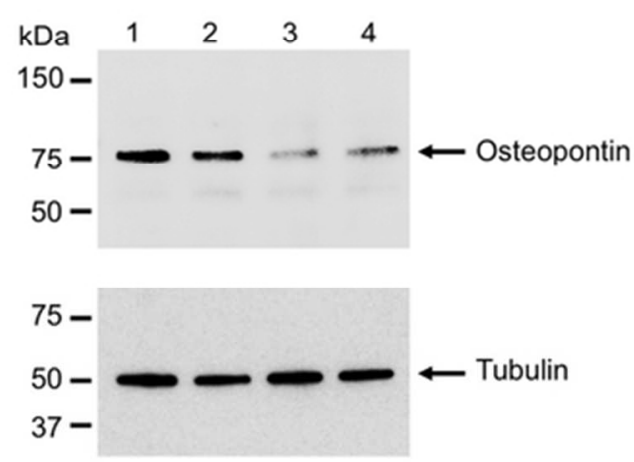

B

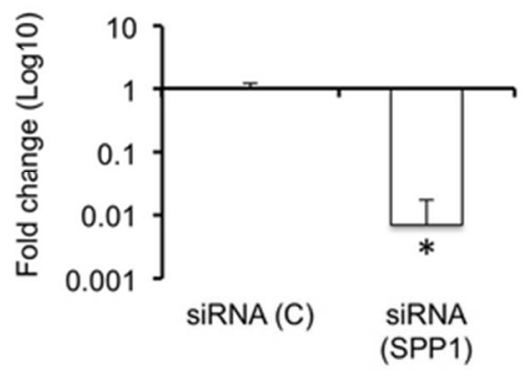

C

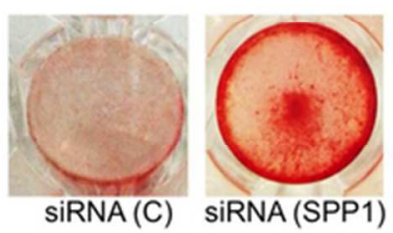

Fig. 5 Effect of osteopontin on the mineralization efficiency of differentiating bone-forming cells isolated from osteoporotic patients. a Equal amounts of protein extracts from hBMSCs incubated for 10 days in growth medium (lane 1) or in osteogenic medium alone (lane 2) or supplemented with $10 \mathrm{nM}$ (lane 3) or $100 \mathrm{nM}$ (lane 4) ZA were loaded onto a SDS-PAGE gel and immunoblotting performed using an antibody specific for osteopontin. Levels of tubulin were used as a loading control. (b) qRT-PCR analysis of SPP1 gene expression in differentiating osteoporotic hBMSCs at day 7 following transfection with either control siRNA (siRNA (C)) or SPP1 siRNA (siRNA (SPP1)). Data was normalized to GUSB and expressed as fold change (Log10) as compared to cells transfected with siRNA (C) using the $2^{-\triangle \Delta C T}$ method. Experiments were performed in triplicate and data expressed as mean \pm SD. * $p<0.01$, in comparison to siRNA (C) as determined by Student's t-test. (c) Representative images of Alizarin red stained osteoporotic hBMSCs transfected with control siRNA (siRNA (C)) or SPP1 siRNA (siRNA (SPP1)) at day 28 post-osteogenic induction.

$56 \times 32 \mathrm{~mm}(300 \times 300$ DPI $)$ 